\title{
A Study on Improvement of Special Acoustic Lens Output through Frequency Trimming
}

\author{
Bong-Young Kim¹, Zhixing Tian ${ }^{2}$ and Myung-Jin Bae ${ }^{3 *}$ \\ ${ }^{1}$ Soong-sil University, Department of Information and telecommunication Engineering, Seoul, 06978, Korea. \\ Orcid Id : 0000-0002-3553-039X \\ ${ }^{2}$ Soong-sil University, Department of Information and telecommunication Engineering, Seoul, 06978, Korea. \\ Orcid Id : 0000-0003-3882-2459 \\ ${ }^{3}$ Soong-sil University, Department of Information and telecommunication Engineering, Seoul, 06978, Korea. \\ Orcid Id : 0000-0002-7585-0400
}

\begin{abstract}
:
The Sound Fire Extinguisher is a device that suppresses flame using sound energy. Therefore, it is important to transmit loud sounds to the flame even under the same conditions. At the Sori Sound Engineering Research Institute (SSERI), sound was focused and resonance and amplification through the special acoustic lens using Horn Effect. Through this, the Sound Fire Extinguisher's high power, small size, and light weight were realized. In this study, we tried to improve output efficiency by efficiently focusing various sound components present in the special acoustic lens through trimming of fundamental frequency. The fundamental wave and higher harmonics in the special acoustic lens cause phase difference due to the length difference of various transmission paths, and interfere with each other to act as reinforcement or extinction. At this time, the phases of composite sounds can be matched through trimming of fundamental frequency. However, it is somewhat unrealistic to match the phases of all sounds on various transmission paths, so we tried to improve the output by finding the optimal frequency where various sounds are efficiently concentrated. In a frequency trimming experiment of $60 \pm 10$ $\mathrm{Hz}$, it was confirmed that the output differed by up to 2.5 times according to the change in frequency. Through these experiments, it was confirmed that the output of the Sound Fire Extinguisher can be improved through frequency trimming using the structural characteristics of the special acoustic lens.
\end{abstract}

Keyword: Sound Fire Extinguisher, special acoustic lens, frequency trimming, phase, output improvement

\section{INTRODUCTION}

The Sound Fire Extinguisher is a device that subjugates fire using sound. In order to control flame with sound energy, very large sound energy must be transmitted to flame. However, the Sound Fire Extinguisher, which was first released by the US Defense Advanced Research Projects Agency (DARPA), was unable to supply energy to the flame smoothly because the sound spread in a spherical shape. For this reason, it was necessary to use a very large speaker of $1000 \mathrm{~W}$ or more to transmit enough energy to extinguish the fire. In addition, it was inevitable to adopt a very large speaker to deliver high power, and power consumption was very large. In order to commercialize the Sound Fire Extinguisher in the actual fire scene, it is necessary to minimize the loss of energy in the route through which the sound is transmitted [1-3].

Sound generally has a transmission characteristic that is intended to spread in all directions. In the case of an open space, since the sound is transmitted from the sound source in a spherical shape, when the distance is double from the sound source, the size of the sound energy per unit area transmitted is reduced to $1 / 4$. It can be said that the level is reduced by $6 \mathrm{~dB}$. Eventually, if the distance to the flame becomes 2 times farther, the power of the Sound Fire Extinguisher must be increased by 4 times. On the other hand, if the sectional area of the route through which the sound is transmitted is kept the same, the sound spread and the energy loss can be minimized [4-5].

The Sori Sound Engineering Research Institute (SSERI) at Soongsil University applied the special acoustic lens to focus sound and minimize the spread of sound in a spherical shape. The special acoustic lens reflects and refracts the sound spreading in a spherical shape by the Horn Effect to focus and form beam-forming. In the study of the special acoustic lens of the existing Sound Fire Extinguisher, the method of collecting sound, resonating and amplifying the sound wave by adjusting the route of the sound wave transmitted inside the horn was adopted. As the special acoustic lens of the Sound Fire Extinguisher amplifies energy using the resonance of the signal, there is room for further efficiency by applying various signal processing technologies. Figure 1 shows the structure of the special acoustic lens applied to the Sound Fire Extinguisher [5-6].

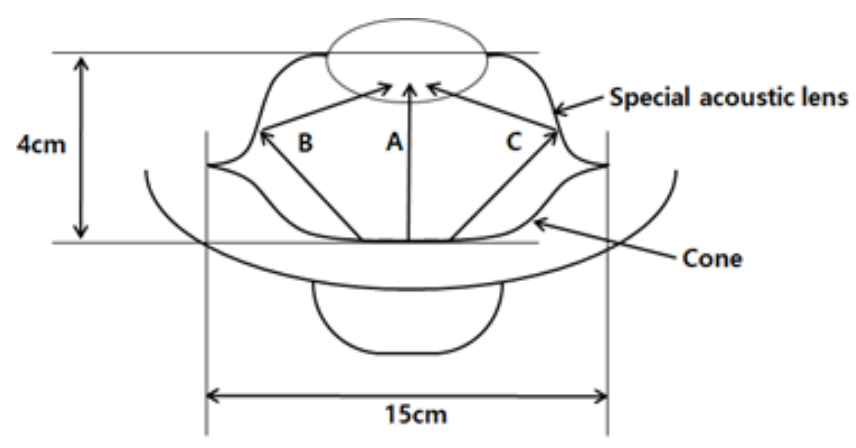

Fig 1. Structure of the Special Acoustic Lens [5] 
In this study, we intend to improve the output efficiency of the special acoustic lens by improving the applied Horn Effect method. The improved method adopted in this study is a conventional method of resonant amplification by focusing phases by converging sounds on various routes, in addition, by matching phases with sound waves that output various higher harmonics generated in the special acoustic lens. It increases resonance amplification efficiency and makes beam-forming more prominent. Chapter 2 describes the resonance amplification method of the Sound Fire Extinguisher special acoustic lens, and Chapter 3 describes the higher harmonics transmitted within the special acoustic lens. Chapter 4 explained how to improve efficiency by matching the phase between the sound wave and higher harmonics proposed in this study. Chapter 5 explains experiments and results, and Chapter 6 concludes.

\section{RESONANCE AMPLIFICATION OF THE SPECIAL ACOUSTIC LENS}

The special acoustic lens applied to the Sound Fire Extinguisher increases the power by focusing and resonating the signal through adjustment of the transmission path of the sound. Figure 2 shows that when the sound of a speaker is concentrated with the special acoustic lens, it resonates and increases power [5].

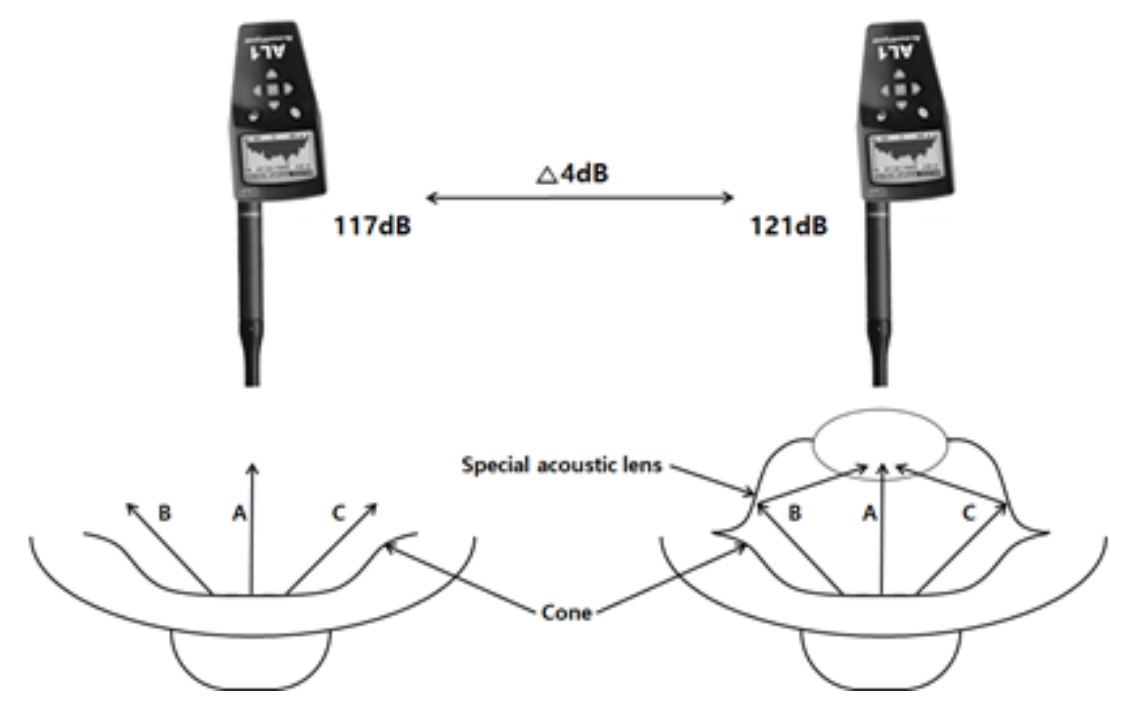

Fig 2. Experiment of Sound Energy Resonance Effect Using the Special Acoustic Lens [5]

The difference in the transmission distance of the collected sound by changing the transmission path of the sound may cause a phase difference. When signals of the same frequency are superpositioned, if the phases are the same, the signals are reinforced and amplified, whereas if the phases are opposite, the signals are interfered and reduced [4-5].

When the phase difference caused by the transmission length difference of sound is $\mathrm{k}_{\mathrm{C}}$, the composite wave $Y(t)$ of two sounds $\left(A \sin \left(\omega t-k_{A}\right), A \sin \left(\omega t-k_{A}-k_{C}\right)\right)$ can be expressed as follows [4][7].

$$
Y(t)=A \sin \left(\omega t-k_{A}\right)+A \sin \left(\omega t-k_{A}-k_{C}\right)
$$

When the frequency of the sound output from the Sound Fire Extinguisher is $60 \mathrm{~Hz}$, and the transmission length difference between the two sounds is $10 \mathrm{~cm}$ within the special acoustic lens, the phase difference $\left(k_{C}\right)$ of the two sounds is very small, $10 / 567 * 2 \pi$. If the two sound levels are the same $(A=B)$, composite wave $Y(t)$ can be expressed as follows [4][7].

$$
\begin{aligned}
Y(t)= & A \sin \left(\omega t-k_{A}\right)+A \sin \left(\omega t-k_{A}-k_{C}\right) \\
= & A \sin \left(\omega t-k_{A}+\frac{k_{C}}{2}\right)+A \sin \left(\omega t-k_{A}-\frac{k_{C}}{2}\right) \\
= & A\left[\sin \left(\omega t-k_{A}\right) \cos \left(\frac{k_{C}}{2}\right)+\cos \left(\omega t-k_{A}\right) \sin \left(\frac{k_{C}}{2}\right)+\sin \left(\omega t-k_{A}\right) \cos \left(\frac{k_{C}}{2}\right)\right. \\
& \left.-\cos \left(\omega t-k_{A}\right) \sin \left(\frac{k_{C}}{2}\right)\right] \\
= & 2 A \sin \left(\omega t-k_{A}\right) \cos \left(\frac{k_{C}}{2}\right)
\end{aligned}
$$


At this time, because the phase difference $k_{c}=10 / 567 * 2 \pi$, it becomes $\cos \left(\frac{k_{C}}{2}\right) \approx 1$, then the composite wave $Y(t)$ can be expressed as follows.

$$
\begin{aligned}
Y(t) & =2 A \sin \left(\omega t-k_{A}\right) \cos \left(\frac{k_{C}}{2}\right) \\
& \approx 2 A \sin \left(\omega t-k_{A}\right)
\end{aligned}
$$

Looking at equation (3), it can be seen that even if there is a transmission length difference between the two sounds, if the phase difference is not large, resonance and amplification are achieved. The special acoustic lens of the Sound Fire Extinguisher focuses low-frequency signals below $100 \mathrm{~Hz}$, thus resonating and amplifying the sound due to the small phase difference occurring on the route.

\section{HIGHER HARMONICS TRANSMITTED WITHIN THE SPECIAL ACOUSTIC LENS}

In order to output a high power sine wave, oscillation, an amplifier, and a heat sink are required, so weighting and large

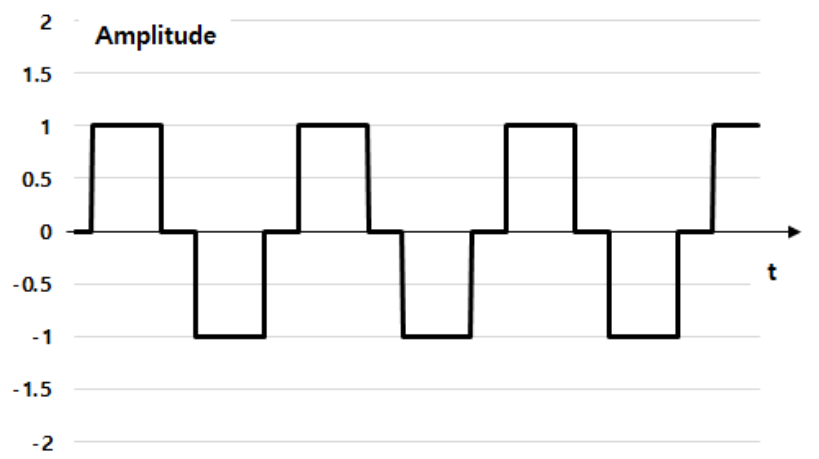

format of the speaker system is inevitable. The Sound Fire Extinguisher applied a Pseudo Sinusoidal Wave rather than a sine wave to realize high power and compact weight while using the existing speaker system. However, unnecessary higher harmonics are generated by outputting the Pseudo Sinusoidal Wave of the FET Pulse Method. Figure 3 is a Pseudo Sinusoidal Wave, and Figure 4 is a Frequency Spectrum that can identify higher harmonics occurring in the Pseudo Sinusoidal Wave [8-9].

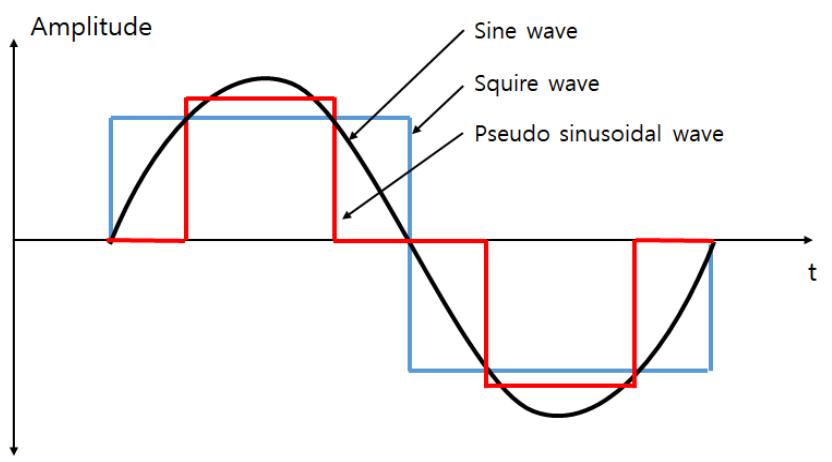

Fig 3. Pseudo Sinusoidal Wave [8-9]

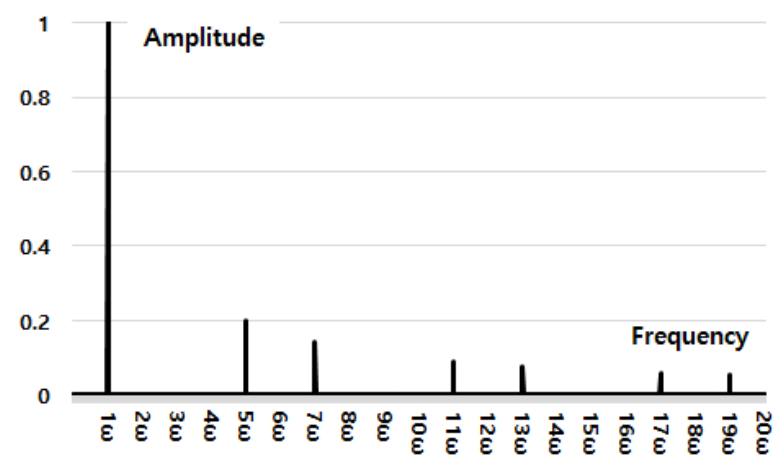

Fig 4. Pseudo Sinusoidal Wave's Frequency Spectrum

Looking at Fig. 4, it can be seen that higher harmonics of integer multiples occur in addition to the fundamental frequency to be output from the Sound Fire Extinguisher. These higher harmonics can be said to be an inevitable output loss caused by outputting the Pseudo Sinusoidal Wave used to achieve high power and miniaturization. However, within the special acoustic lens, the transmission path of higher harmonics can be changed, affecting the resonance and amplification of sounds. In particular, various routes within the special acoustic lens can greatly increase the phase difference between fundamental waves and higher harmonics. Eventually, higher harmonics can be gathered in various sizes and phases through various routes [4][5][7][8].

In addition to higher harmonics caused by fundamental waves, various types of higher harmonics can occur due to distortion due to reflection and refraction inside the special acoustic lens. In these higher harmonics, a phase difference occurs due to a transmission distance difference within the special acoustic lens, and accordingly, resonance and amplification of a signal may be increased or suppressed.

\section{THE SPECIAL ACOUSTIC LENS OUTPUT IMPROVEMENT METHOD THROUGH FREQUENCY TRIMMING}

In this study, we tried to improve the output of the Sound Fire Extinguisher by matching the phases of several sounds with different transmission paths within the special acoustic lens of the Sound Fire Extinguisher. As an application method, for higher harmonics where phase difference is caused by 
transmission path difference, the output is increased by matching the fundamental signal and the peak timing of higher harmonics through trimming of the fundamental frequency. The composite wave between fundamental wave and 7's higher harmonic where phase difference $\left(\theta_{\Delta}\right)$ occurs can be expressed as the following equation.

$$
Y(t)=A_{1} \sin \left(\omega_{1} t\right)+A_{7} \sin \left(\omega_{7} t-\theta_{\Delta}\right)
$$

At this time, the phase difference $\left(\theta_{\Delta}\right)$ is due to the distance difference between sounds transmitted at $340 \mathrm{~m} / \mathrm{sec}$. When the distance difference of the transmitted sound is $d_{\Delta}$, the phase difference can be expressed as the following equation.

$$
\theta_{\Delta}=\frac{d_{\Delta}}{340} X 2 \pi f_{7} \pm 2 \pi N
$$

When the pseudo sinusoidal wave in Figure 3 is output, the amplitude of the 7th higher harmonics corresponds to about $17 \%$ of the fundamental wave. Therefore, the composite wave of the fundamental wave and the 7th higher harmonics in the pseudo sinusoidal wave can be expressed as the following equation.

$$
Y(t)=A_{1} \sin \left(\omega_{1} t\right)+0.17 A_{1} \sin \left(7 \omega_{1} t-\theta_{\Delta}\right)
$$

In order for composite wave $Y(t)$ to produce a peak output, the peak of fundamental frequency and the peak of 7's higher harmonics must match. In the case of $t=\frac{1}{4 f_{1}}$, the fundamental wave becomes the peak. At this time, the following conditions must be met in order for 7's higher harmonics to peak at the same time.

$$
\begin{aligned}
A_{7} \sin \left(\omega_{7} t-\theta_{\Delta}\right) & =0.17 A_{1} \sin \left(7 X 2 \pi f_{1} X \frac{1}{4 f_{1}}-\frac{d_{\Delta}}{340} X 2 \pi X 7 f_{1} \pm 2 \pi N\right) \\
& =0.17 A_{1} \sin \left(\frac{\pi}{2}\right)
\end{aligned}
$$

In order to satisfy equation(7), the fundamental frequency needs to satisfy the following conditions.

$$
f_{1}=(3 \pi \pm 2 \pi N) X \frac{340}{14 \pi d_{\Delta}}
$$

Eventually, when there is a transmission length difference, by adjusting the fundamental frequency, it is possible to match the peak of the seventh higher harmonics with the peak of the fundamental wave.

However, within the special acoustic lens of the Sound Fire Extinguisher, there are various higher harmonics of numerous sounds transmitted through various routes, and the length difference of the transmission path is also inconsistent. Therefore, it can be said that it is effective to find the optimal frequency which all sounds are efficiently concentrated through frequency trimming rather than matching the peaks of all sounds.

The method of frequency trimming is possible by adjusting the switching control signal applied to the Sound Fire Extinguisher. In SSERI, the oscillation and amplification part of the Sound Fire Extinguisher used a FET inverter to output a high power signal by the control signal of low power. At this time, the control signal of low power oscillations the control signal by the $R_{T}$ and $C_{T}$ values of the TL494 chip. In this study, the output frequency of the Sound Fire Extinguisher was trimmed by adjusting the $R_{T}$ of the TL494 chip using variable resistance. Figure 5 is a block diagram of the TL494 chip, and the oscillation frequency $\left(f_{0 s c}\right)$ that can be obtained by adjusting the variable resistance is as shown in Equation (9) [10].

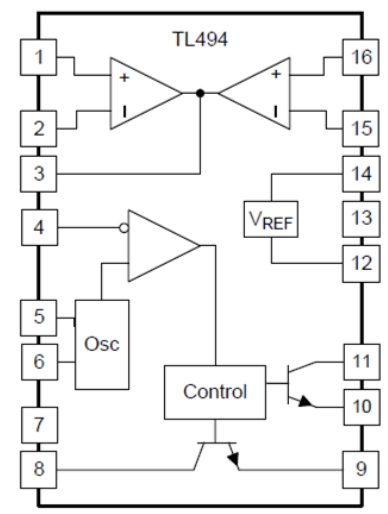

Fig 5. TL494 Block Diagram [10]

$$
f_{\text {OSC }}=\frac{1}{R_{T} \cdot C_{T}}
$$

$$
R_{T}: \text { Timing resistor }
$$

\section{EXPERIMENT AND RESULT}

For the Sound Fire Extinguisher output improvement method proposed in Chapter 4, the effect was confirmed through an experiment to compare the output by trimming the frequency. The method of experiment was to trim the oscillation frequency 
by adjusting the resistance by installing variable resistance on pin 6 of the TL494 chip that oscillations the control signal of the pseudo sinusoidal wave. The frequency range of the fundamental wave output through trimming was $60 \pm 10 \mathrm{~Hz}$. Higher harmonics that transmit various routes within the special acoustic lens are composited with the fundamental wave. At this time, according to the trimming of the frequency, the difference in the sound of the composite wave reinforcement or extinction interference was measured [8][10].
The sound level output from the Sound Fire Extinguisher was measured using NTi Audio's Acoustilyzer AL1. The sound level was measured at a distance of $50 \mathrm{~cm}$ from the Sound Fire Extinguisher. At this time, to minimize the experimental error, all the doors in the laboratory were closed to minimize the influence of external noise. Table 1 shows the results of measuring the Sound Fire Extinguisher output according to trimming, and the measurement results in Table 1 are shown in Figure 6.

Table 1. Output measure result of the Sound Fire Extinguisher according to frequency trimming

\begin{tabular}{|c|c|c|c|c|c|c|}
\hline $\mathrm{f}_{\mathrm{OSC}}(\mathrm{Hz})$ & $\begin{array}{c}\text { Sound level } \\
(\mathrm{dB})\end{array}$ & Deviation & $\mathrm{f}_{\mathrm{OSC}}(\mathrm{Hz})$ & $\begin{array}{c}\text { Sound level } \\
(\mathrm{dB})\end{array}$ & Deviation & Note \\
\hline 50 & 90 & -1.29 & 61 & 92 & 0.71 & \\
\hline 51 & 92 & 0.71 & 62 & 92 & 0.71 & \\
\hline 52 & 91 & -0.29 & 63 & 91 & -0.29 & \\
\hline 53 & 91 & -0.29 & 64 & 91 & -0.29 & \\
\hline 54 & 91 & -0.29 & 65 & 90 & -1.29 & \\
\hline 55 & 93 & 1.71 & 66 & 90 & -1.29 & \\
\hline 56 & 92 & 0.71 & 67 & 90 & -1.29 & \\
\hline 57 & 93 & 1.71 & 68 & 90 & -1.29 & \\
\hline 58 & 93 & 1.71 & 69 & 91 & -0.29 & \\
\hline 59 & 93 & 1.71 & 70 & 89 & -2.29 & \\
\hline 60 & 92 & 0.71 & & & & \\
\hline
\end{tabular}

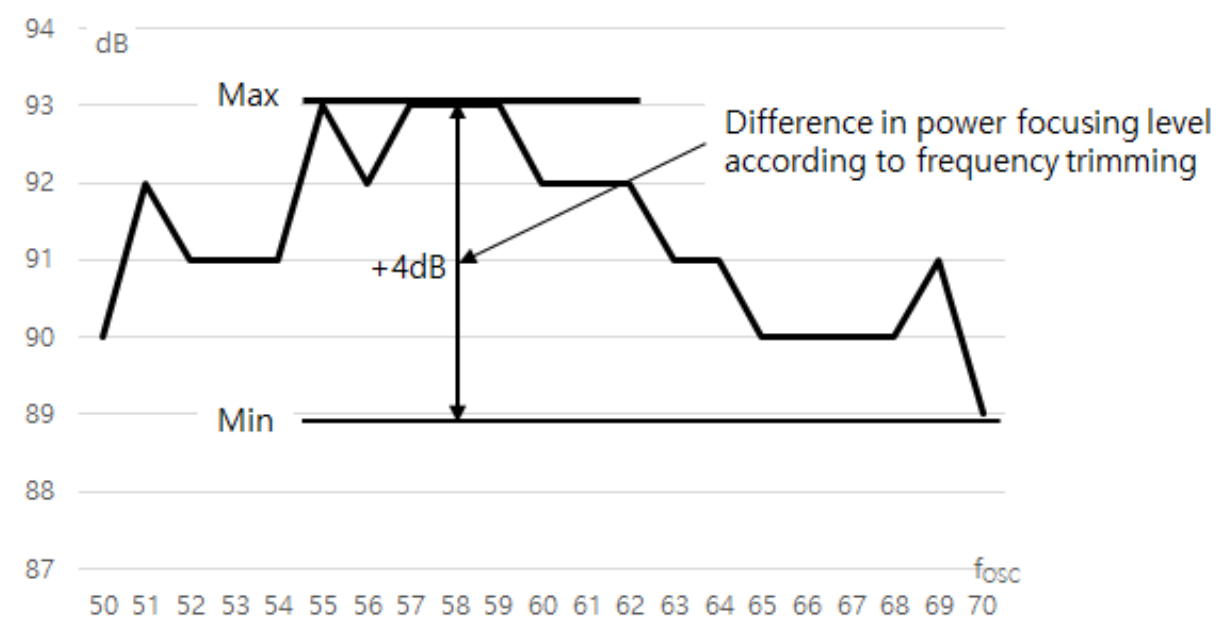

Fig 6. Output comparison according to frequency trimming 
According to the measurement results in Table 1 and Figure 6, the highest power was obtained at $55,57,58$, and $59 \mathrm{~Hz}$ for the Sound Fire Extinguisher used in the experiment. On the other hand, at $70 \mathrm{~Hz}$, the output was relatively small. The output according to frequency trimming showed a maximum difference of $4 \mathrm{~dB}$. This means that the output differs by about 2.5 times depending on the frequency trimming. Through these experiment results, it was confirmed that the output can be improved just by trimming the frequency even at the same power.

\section{CONCLUSION}

The Sound Fire Extinguisher must output a very loud sound to suppress the flame. In SSERI, resonance and amplification were performed by focusing the sound of the Sound Fire Extinguisher by applying the special acoustic lens with the principle of the Horn effect to smoothly transmit sound energy to the flame. In this study, we tried to increase the power of the Sound Fire Extinguisher through frequency trimming for sound components with multiple routes within the special acoustic lens. In the special acoustic lens of the Sound Fire Extinguisher, resonance and amplification can be maximized when the fundamental frequency and higher harmonics components are in phase at the focusing point of the sound. However, in the special acoustic lens, since various higher harmonics have numerous routes, it is effective to find an optimal frequency in which various sounds are efficiently concentrated through frequency trimming.

In the experiment, the fundamental frequency of the Sound Fire Extinguisher was trimmed by adjusting the variable resistance of the TL494 chip. As a result of measuring the special acoustic lens output within the frequency range of $60 \pm 10 \mathrm{~Hz}$, the supplied power was the same, but it appeared at $89 \sim 93 \mathrm{~dB}$ for frequencies, resulting in a difference in output. In particular, it was confirmed that the difference in power occurs up to 2.5 times because $4 \mathrm{~dB}$ occurs between high power and low power. As a result of these experiments, it was confirmed that the output of the special acoustic lens can be improved just by trimming the frequency even at the same power.

\section{REFERENCE}

[1] DARPA 2012.07::

http://www.extremetech.com/extreme/132859-darpacreates-sound-based-fire-extinguisher

[2] Kim, M.-S., Bae, M.-J. "A study on a fire extinguisher with sound focus", Information (Japan), Vol.20, No.6, 2017, pp. 4055-4062.

[3] Yi, E.-Y., Bae, M.-J. "On a fire extinguisher using sound winds", Journal of Engineering and Applied Sciences, Vol.13, No.4, 2018, pp. 977-980.

[4] S. T. Lee. Principles and Application of Sound, Cheong Moon Gak, Korea (2004).

[5] Kim, B.-Y., Bae, M.-J. "A Study on Sound Energy Resonance Using the Special Acoustic Lens", International Journal of Engineering Research and Technology, Vol.12, No5, 2019, pp. 642-646.

[6] Kim, B.-Y., Bae, M.-J. "A Study on Sound Beam Formation of Sound Fire Extinguisher", International Journal of Engineering Research and Technology, Vol.12, No7, 2019, pp. 1014-1019.

[7] Kim, B.-Y. "A Study on Performance Improvement of the Sound Fire Extinguisher", Soongsil University Doctoral thesis, 2019.12.

[8] Kim, B.-Y., Bae, M.-J. "A Study on High Power Amplifier of Sound Fire Extinguisher Using Pseudo Sinusoidal Wave", International Journal of Engineering Research and Technology, Vol.12, No7, 2019, pp. 11331137.

[9] W. Farrer and J.D. Miskin, "Quasi-sine-wave fully regenerative invertor", Proceedings of the Institution of Electrical Engineers, Volume 120, Issue 9, September 1973, p. $969-976$

[10] TL494 Pulse-Width-Modulation Control Circuits, Texas Instruments Incorporated, 2018

[11] D. G. Cho, "(A)novel structure of vertical power MOSFET with improved switching speed", Thesis for Master`s Degree, Sogang University (2013).

[12] George Mason University youtube: https://www.youtube.com/watch?v=uPVQMZ4ikvM .

[13] Boffard, R., "Burning issue", Engineering and Technology, Vol.10, No.7, 2015, pp. 48-51. 